\title{
EDITORIAL
}

\section{THE ORDEALS OF THE LONG HAULERS: FACING AND TRACING THE AFTERMATH OF COVID-19}

\section{Md Robed Amin}

The long haulers of the COVID-19 infection are distinct, cluster of symptoms and majority prolongs from acute to chronic sufferings and the course of this symptoms are commonly prolonged with few of them leads to catastrophe and disability. "The Long Haulers" are also known as "Longings" or lingering symptoms as described by $\mathrm{WHO}^{1}$. The common features of covid-19 infection are if recovery happens, it usually is completed within 2 to 6 weeks $^{2}$. For some people, some symptoms may linger or recurs after the 6 weeks duration of time following initial recovery. This can also happen in mild disease and people are noninfectious in this period. World Health Organization China Joint Mission on COVID-19 suggested that the median time from onset to clinical recovery for mild cases is approximately 2 weeks and is 3-6 weeks for patients with severe or critical disease ${ }^{1}$

There has been lack of scientific study on this longing initially as the physicians and scientist were busy with acute case management in COVID-19 during the volcano of pandemic. That's why the patients group themselves motivated to start a telephone survey in USA to address the issue of longings. The survey was later published to observe the long symptoms and focused in CDC as well ${ }^{3}$
Since then, there has been growing awareness of the long-term impacts of COVID-19 infection, including cardiac, neurological, metabolic and respiratory longterm sequelae ${ }^{2}$. There has been observed of these symptoms even in young adults and children without underlying medical conditions. There are many case reports from people where they did not get back to their previous well being following COVID-19. Little is known about the clinical course after the mild illness. The telephonic survey revealed that 35\% cases were not able to return to their previous work even after 2 to 3 weeks of the apparent recovery ${ }^{3}$. Among those who were 18 to 34 years of old with good health, one out of five reports that few features were prolonged. The report also revealed that obesity, hypertension and previous mental health was the risk factors of getting the longings.

The physiologic and pathological spectrum of respiratory system in COVID-19 infection after the $6^{\text {th }}$ week and few postmortem studies with diffuse alveolar damage presumed that the prolonged respiratory illness in the form of interstitial lung disease is possible $4,5,6,7$. HRCT of lung study of 165 patients with COVID-19 and follow-up information, Jin et al. [not peer-reviewed] reported $13.9 \%(23 / 165)$ showed diffuse

\section{Lingering symptoms reported by participants of a multi-state phone study in the USA}
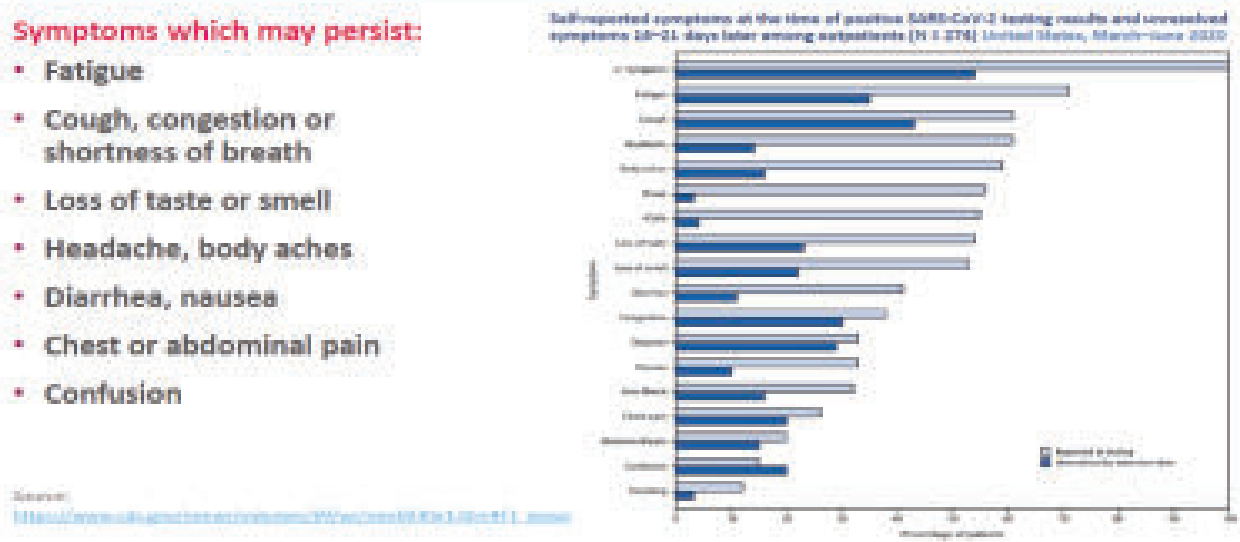

Professor of Medicine, Dhaka Medical College

Bangladesh J Medicine 2021; 32 : 1-3

DOI: https://doi.org/10.3329/bjm.v32i1.51087 
alveolar damage In follow-up CTs performed e"30 days after symptom onset, $22.0 \%(9 / 41)$ of patients still showed diffuse alveolar damage ${ }^{8}$.

The expression of ACER 2 in the myocyte and abundant of receptor in cardiovascular system precludes the possibilities of cardiovascular sequalae is expected longings of COVID-19 infection. Acute coronary syndrome, myocardial infarction, arrhythmia, cardiogenic shock, heart failure, myocarditis and venous thromboembolism were listed as presumptive possibilities suggested by driggins et al. MIS-C is a newly recognized illness that may be associated with SARS-CoV-2. Reports suggest that MIS-C appears weeks after potential infection with or exposure to COVID-19(.26-28) Symptoms are variable, but many children have symptoms similar to toxic shock syndrome or Kawasaki disease. Feldstein et al. reported on patients with MIS-C patients from 26 states in the United States (US) Most patients required intensive care unit (ICU) admission (80\%, 148/186), 20\% required mechanical ventilation (37/186), 8\% (15/186) had coronary artery aneurysms and three children died. The median age of patients in the study was 8.3 years (IQR: 3.3.-12.5). Dufort et al. reported 99 confirmed and suspected cases in new york. Most patients required admission to an ICU (80\%, 79/99), $10 \%$ (10/99) required mechanical ventilation, 9\% (9/ 99) had coronary artery aneurysms and two patients died. $42 \%$ (42/99) of patients were 6-12 years old .

There are few studies to date documenting long-term neurological sequelae related to COVID-19. Numerous studies investigated the long-term persistence of sensory deficits specifically on olfactory (smell) and gustatory (taste) dysfunction. These studies (11 to 16) of different observation revealed that there is some evidence of olfactory dysfunction (e.g., dysosmia) and gustatory dysfunction (e.g., dysgeusia) as relatively common long-term sequelae associated with COVID19 infection $^{11,12}$. Macro thrombosis in the internal carotid artery in patients with mild respiratory symptoms of COVID-19 \& Stroke as a late presenting symptom of the disease. A multicentre series of 26 patients with COVID-19 and either ischaemic or haemorrhagic events reported that $27 \%$ were younger than 50 years.

Myalgic encephalomyelitis / chronic fatigue syndrome (ME/CFS) are debilitating \& poorly understood condition associated with COVID-1913. Lasting fatigue worsens after exercise or mental exertion, inability to concentrate or "Brain fog". It's a pathological process with inflammation of brain and spinal cord and elevated inflammatory markers, cytokine in spinal fluid.(Japanese study), overactive immune system, low grade encephalitis, epigenetic change in gene expression \& downstream metabolic alterations were observed. MRI of brain based follow up study showed recovered COVID-19 can exhibit neurological symptoms. MRI brain shows wide variety of microstructural changes to several areas. Other types of COVID-19 family can cause neurodegeneration, demyelination, \& other neurological damage. 63\% showed neurological symptoms in acute COVID-19. Three month later, there are still have symptoms in $55 \%$ cases in the form of mood change, fatigue, headache. DTI MRI scan showed some ongoing neurological damage by virus in olfactory cortices,insulas, hippocampi,left Rolandic\& right cingulate gyrus.Right hemisphere was more possibly due to olfactory function in right brain. Severity of illness has little effect even mild one is affected ${ }^{14}$.

Renal and hepatic long term sequalae was also observed scarcely in few literatures. Adapa et al. report AKI during COVID-19 infection, which could lead to potential long-term impacts ${ }^{15}$. Wang et al. reported that COVID-19 does not result in AKI based on a study that included 116 hospitalized patients in China with a median age of 54 years (IQR: 38-69) ${ }^{16}$. In a metaanalysis, the pooled prevalence of liver injury in 4,191 people with COVID-19 was 19.5\% (95\% CI: 14.3-26.1) with substantial variations among the 19 studies (Samidoust et al.) Given the potential for liver injury during COVID-19 infection, it is not unreasonable to assume long-term hepatic sequelae may occur in recovered patients

Little is currently known about the long-term reproductive sequelae associated with COVID-19 infection; however, there is one study that suggests that sperm quantity and function may be impacted. A review indicates decreased sperm concentration and motility for up to 90 days following COVID-19 infection (Segars et al.)

COVID-19 can result in prolonged illness and persistent symptoms even in young adults and who does not have previous medical conditions and even in patient who were not admitted in hospital. Much is still, known how the COVID-19 can affect human in prolonged time. More time and research is needed to understand the long term effect of COVID-19, why symptoms persist or recur, how these health problems affect patients, clinical course and likelihood recovery. Effective public health messaging is also needed for young adults. Monitoring social media (e.g facebook, tweets, Instagram etc) on long term effect of COVID19 is needed as the most discussed topic in 2020 in social medica was long haulers of COVID- 19.Among $11 \%$ of all conversation in social medica, the 'long covid' was used repeatedly. 
Longings in covid-19 both acute and prolonged is common. Underlying pathophysiology remains to be totally determined. All areas of the systems are involved with various order in various time frame. Multiple comorbidities pose to more severe longings in Covid19 and some are prolonged. Inter specialty collaboration and innovative thinking will render quality and appropriate health care services. COVID19 is a disease that primarily affects the lung, that can impact any part of the body including any system.

\section{References:}

1. World Health Organization (WHO). Report of the WHOChina Joint Mission on Coronavirus Disease 2019 (COVID-19): 16-24 February 2020 [Internet]. Geneva: World Health Organization; 2020 [cited 2020 Jul 06]. Available from: https://www.who.int/docs/defaultsource/coronaviruse/who-china-joint-mission-oncovid-19-final-report.pdf

2. Dasgupta A, Kalhan A, Kalra S. Long term complications and rehabilitation of COVID-19 patients. JPMA J Pak Med Assoc. 2020;70(Suppl 3)(5):S131-S5. Available from: https://doi.org/10.5455/JPMA.32

3. http; www.cdc.gov/mmwr/volume /69/wr/mm6930 e1/htmf 1

4. https://www.ncbi.nlm.nih.gov/pmc/articles/ PMC7192220/

5. https://jamanetwork.com/journals/jamainterna lmedicine/fullarticle/415378

6. Carsana L, Sonzogni A, Nasr A, Rossi RS, Pellegrinelli A, Zerbi $\mathrm{P}$, et al. Pulmonary post-mortem findings in a series of COVID-19 cases from northern Italy: a twocentre descriptive study. Lancet Infect Dis. 2020 Jun 08 [Epub ahead of print]. Available from: https:// doi.org/10.1016/s1473-3099(20)30434-5

7. Schaller T, Hirschbühl K, Burkhardt K, Braun G, Trepel $\mathrm{M}$, Märkl B, et al. Postmortem examination of patients with COVID-19. JAMA. 2020;323(24):2518-20. Available from: https://dx.doi.org/10.1001\%2Fjama. 2020.8907

8. Jin C, Wang Y, Wu CC, Zhao H, Liang T, Liu Z, et al. A pattern categorization of $\mathrm{CT}$ findings to predict outcome of COVID-19 pneumonia. medRxiv 20107409 [Preprint].
2020 May 19 [cited 2020 Jul 06]. Available from: https:/ /doi.org/10.1101/2020.05.19.20107409

9. Driggin E, Madhavan MV, Bikdeli B, Chuich T, Laracy J, Biondi-Zoccai G, et al. Cardiovascular considerations for patients, health care workers, and health systems during the COVID-19 pandemic. J Am Coll Cardiol. 2020;75(18):2352-71. Available from: https://doi.org/ 10.1016/j.jacc.2020.03.031

10. Sardari A, Tabarsi P, Borhany H, Mohiaddin R, Houshmand G. Myocarditis detected after COVID-19 recovery. Eur Heart J Cardiovasc Imaging. 2020 May 27 [Epub ahead of print]. Available from: https:// doi.org/10.1093/ehjci/jeaa166

11. Sayin I, Yazici ZM. Taste and smell impairment in SARSCoV-2 recovers early and spontaneously: experimental data strongly linked to clinical data. ACS Chem Neurosci. 2020 Jun 15 [Epub ahead of print]. Available from: https://doi.org/10.1021/ acschemneuro.0c00296

12. Lee Y, Min P, Lee S, Kim SW. Prevalence and duration of acute loss of smell or taste in COVID-19 patients. J Korean Med Sci. 2020;35(18):e174. Available from: https://doi.org/10.3346/jkms.2020.35.e174

13. Solomon IH, Normandin E, Bhattacharyya S, et al. Neuropathological features of Covid-19. N Engl J Med. DOI: 10.1056/NEJMc2019373 June 12, 2020

14. Neurological manifestations of hospitalized patients with COVID-19 in Wuhan, China: a retrospective case series study. Mao L, Wang $M$, Chen $S$, et al. medRxiv. 2020

15. Adapa S, Chenna A, Balla M, Merugu GP, Koduri NM, Daggubati SR, et al. COVID-19 pandemic causing acute kidney injury and impact on patients with chronic kidney disease and renal transplantation. J Clin Med Res. 2020;12(6):352-61. Available from: https:// doi.org/10.14740/jocmr4200 Long-Term Sequelae and COVID-19 - What We Know So Far 10

16. Wang L, Li X, Chen H, Yan S, Li D, Li Y, et al. Coronavirus disease 19 infection does not result in acute kidney injury: an analysis of 116 hospitalized patients from Wuhan, China. Am J Nephrol 2020;51(5):343-8. Available from: https://doi.org/ $10.1159 / 000507471$ 